\title{
Calibrating the ink spreading curves enhanced Yule-Nielsen modified spectral Neugebauer model with the two-by-two dot centering printer model
}

\author{
R.Rossier, R. D. Hersch \\ School of Computer and Communication Sciences \\ Ecole Polytechnique Fédérale de Lausanne (EPFL), Switzerland
}

\begin{abstract}
The Yule-Nielsen modified spectral Neugebauer model enhanced for accounting for ink spreading in the different ink superposition conditions (EYNSN) requires measuring the reflectances of halftone calibration patches in order to compute the ink spreading curves mapping nominal ink surface coverages to effective ink surface coverages. Spectral measurements of dozens of halftone patch reflectance spectra for each considered halftoning method, screen shape or screen frequency variant is cumbersome and time consuming. As an alternative, we compute automatically the reflectance of the calibration patches necessary to establish the ink spreading curves by making use of the two-by-two dot centering model (abbreviated "2-by-2"). The two-bytwo dot centering model requires its own set of calibration patches, among which a very small subset is necessary for predicting the reflectance of the EYNSN calibration patches. For a given combination of printer, paper and inks, using only 68 two-by-two calibration patches for the three CMY inks, respectively 118 twoby-two calibration patches for the four CMYK inks, we are able to predict the reflection spectra of most classical halftone screens and at many different frequencies. We compare the results of predictions where the EYNSN model calibration patches are measured or are predicted with the two-by-two dot centering model. For 125 (CMY) and 625 (CMYK) uniformly distributed patches printed with an ink-jet printer at three different screen frequencies (75lpi, 1001pi and 125lpi), the mean reflectance prediction accuracy is similar, i.e. the decrease in accuracy due to the two-by-two prediction of EYNSN calibration patch reflectance is less than $\Delta E_{94}=0.4$.
\end{abstract}

Keywords: color reproduction, ink spreading model, spectral prediction model, Yule-Nielsen modified Spectral Neugebauer, halftones, ink spreading curves, dot gain, ink superposition conditions, two-by-two dot centering model

\section{INTRODUCTION}

Many different phenomena influence the reflection spectrum of a color halftone patch printed on a diffusely reflecting substrate (e.g. paper). These phenomena comprise the surface (Fresnel) reflection at the interface between the air and the print; light scattering and reflection within the substrate (i.e. the paper bulk); and the internal (Fresnel) reflections at the interface between the print and the air. The lateral scattering of light within the paper substrate and the internal reflections at the interface between the print and the air are responsible for what is generally called optical dot gain, also known as the Yule-Nielsen effect.

Due to the printing process, the deposited ink dot surface coverage is generally larger than the nominal surface coverage, yielding a "physical" dot gain responsible for the ink spreading phenomenon [1]. Effective ink dot surface coverages depend on the inks, on the paper, and also on the specific superposition of an ink halftone and other inks. At the present time, according to the literature [1], [2], [3], among the existing spectral reflection prediction models, mainly the well-known Yule-Nielsen modified spectral Neugebauer model (YNSN) [5], [6] is used for predicting reflection spectra. This model is further enhanced by accounting for the ink spreading phenomenon (denomination: EYNSN) [4]. Ink spreading is computed by taking into account the respective physical dot gains of one ink halftone printed in different superposition conditions, i.e. 
alone on paper, in superposition with one ink, in superposition with two inks and in superposition with three inks. Effective dot surface coverages are fitted separately for every superposition condition by minimizing a difference metric between measured reflection spectrum and predicted reflection spectrum. This yields, for each superposition condition, an ink spreading curve mapping nominal to effective surface coverages [4]. To predict the spectral reflectance of a color halftone, nominal surface coverage values are converted into effective coverage values by weighting the contributions of the different ink spreading curves according to the ratios of colorant surface coverages. Since ink spreading curves are created for a specific halftoning screen shape and screen frequency, this type of predictions is halftone-algorithm dependant. Calibration of dot surface coverages performed for one halftone algorithm can not be used by another algorithm without loosing prediction accuracy.

More than ten years ago, Shen-ge Wang proposed a "tiling" method capable of characterizing a black and white [7] or a color [10] print. Since printed dots are not perfect squares and adjacent dots overlap each other, he proposed a set of two-by-two patterns which account for the contribution of the neighbourhood printed dots. When carrying out the tiling of a given halftone and predicting its reflectance according to the YuleNielsen modified Neugebauer model, the two-by-two model requires a calibration set of 1072 patterns [10] in the case of a CMY print. This two-by-two dot centering spectral prediction method is halftone-algorithm independent, i.e. the initial calibration can be used for predicting the reflectances of halftone prints having dot screens of various frequencies and shapes.

In the present contribution, we reduce the calibration effort required by the EYNSN model by predicting with the two-by-two dot centering model the calibration patches enabling creating the ink spreading curves. Using 68 patterns in the case of a 3 ink print, respectively 116 in the case of a 4 ink print; we create by computation the ink spreading curves for the selected halftoning method, screen shape and screen frequency. The conjunction of the two-by-two dot centering concept and of the ink spreading enhanced Yule-Nielsen spectral Neugebauer model eliminates the need to calibrate the two-by-two dot centering model with 1072 patches in case of three inks and 16576 patches in case of four inks but still guarantees the advantages of a halftonealgorithm independent spectral or color prediction model.

\section{INK SPREADING CURVES ENHANCED YULE-NIELSEN MODIFIED SPECTRAL NEUGEBAUER MODEL (EYNSN)}

The ink spreading model accounting for ink spreading in all ink superposition conditions [4] relies on ink spreading curves mapping nominal surface coverages to effective surface coverages for (a) the surface coverages of single ink halftones, (b) the surface coverages of single ink halftones superposed with one solid ink and (c) the surface coverage of single ink halftones superposed with two solid inks. In order to obtain the effective coverages $\left(c^{\prime}, m^{\prime}, y^{\prime}\right)$ of a color halftone as a function of its nominal surface coverages $(c, m, y)$, the contributions of the different ink spreading curves are weighted according to the ratio of colorants forming that halftone.

During calibration of the model, the ink spreading curves of single ink halftones printed in superposition with paper white, with one solid ink or with two solid inks are obtained by measuring the reflection spectra $R(\lambda)$ at $25 \%, 50 \%$ and $75 \%$ nominal surface coverages and by fitting effective surface coverages using the YuleNielsen modified spectral Neugebauer model (YNSN) [6]

$$
R(\lambda)=\left(\sum_{i} a_{i} R_{i}(\lambda)^{1 / n}\right)^{n}
$$

where $n$ is the surface coverage, $R_{\mathrm{i}}$ is the reflection spectrum of $i^{\text {th }}$ colorant (also called Neugebauer primary) and $n$ is an empirical scalar coefficient related to the halftone screen frequency $(1<n<10)$. 
We obtain the ink spreading curves mapping nominal to effective surface coverages for each ink, in each ink superposition condition, by linear interpolation between the obtained effective surface coverages,.

For cyan, magenta and yellow inks with nominal coverages $c, m$ and $y$, the ink spreading functions (curves) mapping nominal coverages to effective coverages for single ink halftones are $f_{c}(\mathrm{c}), f_{m}(m)$ and $f_{y}(y)$. The functions mapping nominal coverages of an ink to effective coverages of that ink, for single ink halftones superposed with a second solid ink and for single ink halftones superposed with two solid inks are respectively $f_{c / m}(c), f_{c / y}(c), f_{m / c}(m), f_{m / y}(m), f_{y / c}(y), f_{y / m}(y)$ and $f_{c / m y}(c), f_{m / c y}(m), f_{y / c m}(y)$, where $f_{i j /}(i)$ indicates an ink halftone $i$ superposed with solid ink $j$, and where $f_{i j k}(i)$ indicates an ink halftone $i$ superposed with solid inks $j$ and $k$. In the case of three inks, these 12 functions may for example be obtained by fitting 36 patches, i.e. 3 patches $(25 \%, 50 \%$ and $75 \%$ nominal surface coverages) per function. These functions may also be obtained using only the effective surface coverage at $50 \%$ nominal surface coverage.

In order to obtain the effective surface coverages $c^{\prime}, m^{\prime}$ and $y^{\prime}$ of a color halftone patch, it is necessary, for each ink, to weight the contributions of the corresponding ink spreading curves. For example, for deducing the cyan ink halftone surface coverage, we need to weight the contributions of the curves $f_{c}, f_{c / m}, f_{c / y}$ and $f_{c / m y}$. The weighting functions depend on the effective surface coverages of the colorants on which the considered ink halftone is superposed. Let us assume that inks are printed independently of each other. For the considered system of 3 inks cyan, magenta and yellow with nominal coverages $c, m$ and $y$ and effective coverages $c^{\prime}, m^{\prime}$ and $y^{\prime}$, the equations (2) rely on the relative weight, i.e. the relative surface of the underlying colorants.

In analogy with Demichel's equations (3), the relative weight of the underlying white colorant (cyan superposed with colorant paper only) is (1-m') (1-y'), the underlying magenta colorant (cyan superposed with colorant magenta only) is $m^{\prime}$ (1-y'), the underlying yellow colorant (cyan superposed with colorant yellow only) is (1-m') $y^{\prime}$ and the underlying red colorant (cyan superposed with both solid magenta and yellow) is $m ' y$ '. The resulting system of equations is [4]:

$$
\begin{aligned}
& c^{\prime}=f_{c}(c)\left(1-m^{\prime}\right)\left(1-y^{\prime}\right)+f_{c / m}(c) m^{\prime}\left(1-y^{\prime}\right)+f_{c / y}(c)\left(1-m^{\prime}\right) y^{\prime}+f_{c / m y}(c) m^{\prime} y^{\prime} \\
& m^{\prime}=f_{m}(m)\left(1-c^{\prime}\right)\left(1-y^{\prime}\right)+f_{m / c}(m) c^{\prime}\left(1-y^{\prime}\right)+f_{m / y}(m)\left(1-c^{\prime}\right) y^{\prime}+f_{m / c y}(m) c^{\prime} y^{\prime} \\
& y^{\prime}=f_{y}(y)\left(1-c^{\prime}\right)\left(1-m^{\prime}\right)+f_{y / c}(y) c^{\prime}\left(1-m^{\prime}\right)+f_{y / m}(y)\left(1-c^{\prime}\right) m^{\prime}+f_{y / c m}(y) c^{\prime} m^{\prime}
\end{aligned}
$$

This system of equations can be solved iteratively: one starts by setting initial values of $c^{\prime}, m^{\prime}$ and $y^{\prime}$ equal to the respective nominal coverages $c, m$ and $y$. After one iteration, one obtains new values for $c^{\prime}, m^{\prime}$ and $y^{\prime}$. These new values are used for the next iteration. After a few iterations, typically 4 to 5 iterations, the system stabilizes and the obtained coverages $c^{\prime}, m^{\prime}$ and $y^{\prime}$ are the effective coverages. The system of equations (2) yields the effective surface coverages of cyan, magenta and yellow inks for the corresponding nominal surface coverages.

The effective colorant coverages are obtained from the effective coverages of the inks according to the Demichel equations (3) which give the respective surface coverages of the colorants as a function of the surface coverages of the individual inks. In case of independently printed cyan, magenta and yellow inks of respective surface coverages $c^{\prime}, m^{\prime}, y^{\prime}$, the respective fractional areas of the colorants white, cyan, magenta, yellow, red (superposition of magenta and yellow), green (superposition of yellow and cyan), blue (superposition of magenta and cyan) and black (superposition of cyan, magenta and yellow) are : 


$\begin{array}{ll}\text { White } & a_{{ }^{\prime}}=\left(1-c^{\prime}\right)\left(1-m^{\prime}\right)\left(1-y^{\prime}\right) \\ \text { Cyan } & a_{c}^{\prime}=c^{\prime}\left(1-m^{\prime}\right)\left(1-y^{\prime}\right) \\ \text { Magenta } & a_{{ }_{m}}^{\prime}=\left(1-c^{\prime}\right) m^{\prime}\left(1-y^{\prime}\right) \\ \text { Yellow } & a_{{ }_{y}}^{\prime}=\left(1-c^{\prime}\right)\left(1-m^{\prime}\right) y^{\prime} \\ \text { Red } & a_{r}^{\prime}=\left(1-c^{\prime}\right) m^{\prime} y^{\prime} \\ \text { Green } & a_{{ }_{g}}^{\prime}=c^{\prime}\left(1-m^{\prime}\right) y^{\prime} \\ \text { Blue } & a_{{ }_{b}}^{\prime}=c^{\prime} m^{\prime}\left(1-y^{\prime}\right) \\ \text { Black } & a_{k}^{\prime}=c^{\prime} m^{\prime} y^{\prime}\end{array}$

The complete model accounting for ink spreading in all superposition conditions is illustrated in Figure 1. The $n$-value of the Yule-Nielsen modified spectral Neugebauer model for a given printer and screen element frequency is obtained by computing for a subset of the considered color samples the mean CIELAB $\triangle E 94$ color difference between predicted and measured reflection spectra. By iterating across possible $n$-values, one selects the $n$-value yielding the lowest color difference between predicted and measured reflection spectra.

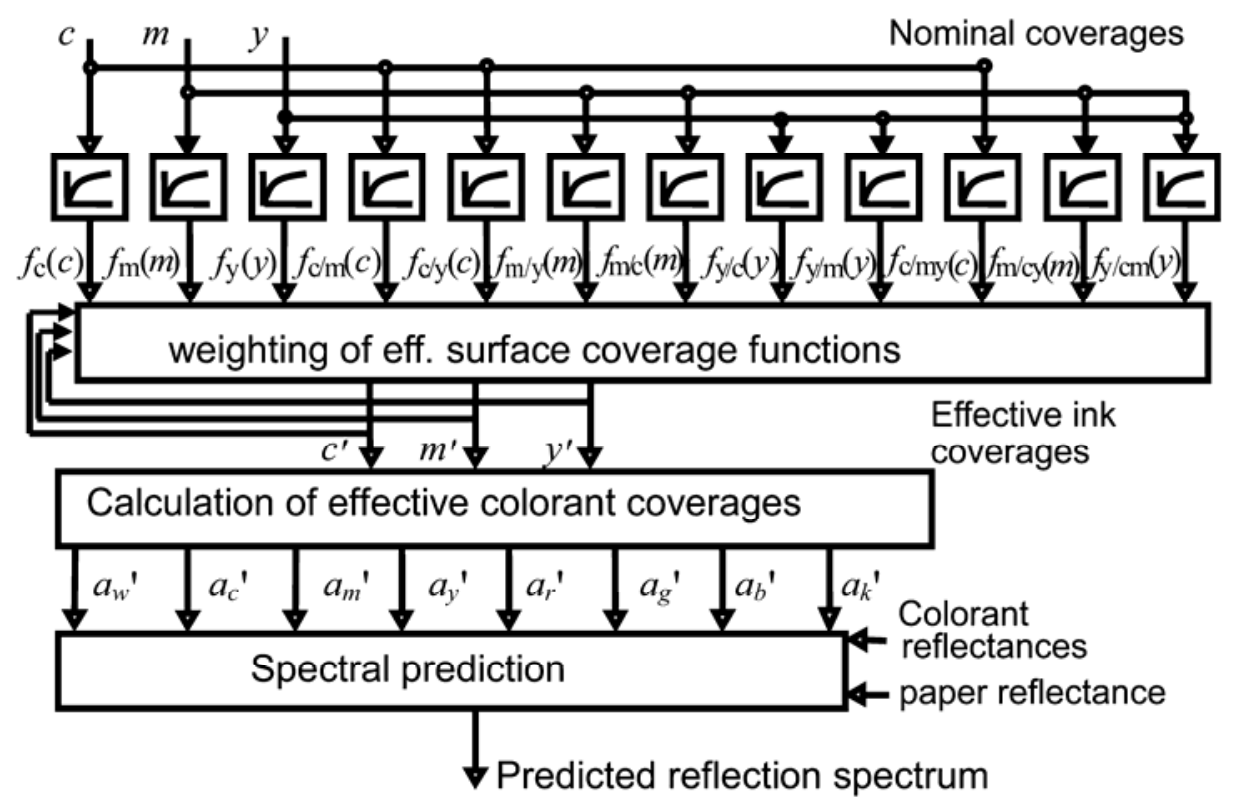

Fig. 1. Prediction model with ink spreading in all superposition conditions.

\section{PREDICTING THE EYNSN CALIBRATION PATCH REFLECTION SPECTRA}

According to the two-by-two dot centering model [7], [8], [9], in order to characterize black and white halftones, there is a need of only seven independent patterns. These seven patterns together with their symmetrical instances along the horizontal and vertical axes describe all possible black/white distributions within a 2-by-2 pixel tile. Symmetrical patterns generate identical patterns in shape and ink surface coverage. Figure 2 (a) illustrates the seven 2-by-2 calibration patterns G0 to G6. In order to create full patches, these bitmap are replicated and sent to the printer. Their reflectance spectra are measured with a spectrophotometer. Then, these patterns are mapped into the halftone whose reflectance is to be predicted. We compute the number of occurrences of each 2-by-2 pattern (G0...G6) or their symmetrical counterparts appearing within that halftone bitmap, see Figure 2 (b). 


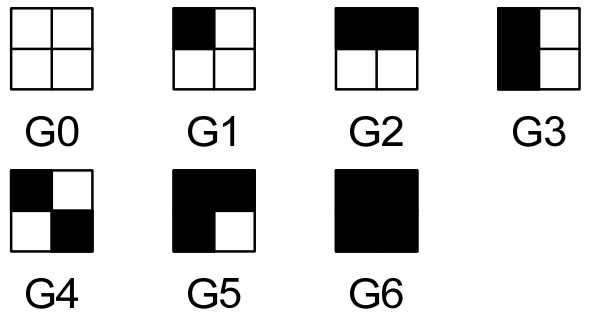

(a)

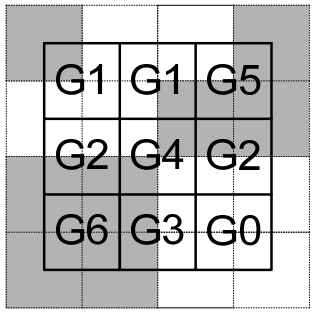

(b)

Fig. 2. (a) The 7 calibration patterns for the black and white 2-by-2 model. (b) Example of a halftone with the corresponding mapped 2-by-2 patterns.

Finally, the predicted halftone reflectance spectrum $R$ is computed using Equation (4), where $i_{m}$ is the number of occurrences of output calibration pattern $G_{m}$ mapped into the halftone and $R_{m}$ is its corresponding measured reflection spectrum

$$
R^{1 / n}=\sum_{m=0}^{6} i_{m} \cdot R_{m}^{1 / n} / \sum_{m=0}^{6} i_{m}
$$

The 2-by-2 dot centering model can be extended from black and white printing to color printing [10]. Since the dots within a 2-by-2 tile have more than the two black and white colors, the number of patterns that can occur within a 2-by-2 tile increases significantly. The number of possible arrangements for the four printed dots of a 2-by-2 tile and for $N$ solid colorants is $N^{4}$. In the case of a CMY print (8 colorants), there are $8^{4}=4096$ possible colors arrangements. If we remove both the horizontal and the vertical symmetries, 1072 independent patterns remain. For $N$ colorants, the total number of independent patterns $P(N)$ is given by Equation (5), see [11]

$$
P(N)=\left\{\begin{array}{c}
\frac{N !}{(N-2) ! \cdot 2} 5+\frac{N !}{(N-1) !} \quad N=2 \\
\frac{N !}{(N-4) ! \cdot 4 !} 6+\frac{N !}{(N-3) ! \cdot 3 !} 9+\frac{N !}{(N-2) ! \cdot 2} 5+\frac{N !}{(N-1) !} \quad N>3
\end{array}\right.
$$

For black and white printings there are $\mathrm{P}(2)=7$ independent patterns and for CMY printings, there are $\mathrm{P}(8)=1072$ independent patterns. The two-by-two dot centering model becomes untractable for CMYK printings because we need $\mathrm{P}(16)=16576$ independent patterns. If we rewrite Equation (4) for the case of color predictions with $N$ solid colorants, we obtain Equation (6), see [10]:

$$
R^{1 / n}=\sum_{m=0}^{P(N)-1} i_{m} \cdot R_{m}{ }^{1 / n} / \sum_{m=0}^{P(N)-1} i_{m}
$$

When calibrating the EYNSN for a CMY print, we create for each halftone printed alone or in superposition with one or two solid ink (12 possible superposition conditions) a function mapping nominal coverages to effective coverages. For the calibration of the EYNSN model, we need the reflection spectra of 8 solid colorants (Neugebauer primaries: white, cyan, magenta, yellow, red, green, blue, black) and of 12 halftones superposed either on paper, on one ink or on two inks. Each of the 12 calibration halftones is composed of 5 possible 2-by-2 patterns (G1 to G5). In total, the EYNSN calibration requires therefore $12 \cdot 5+8=68$ patches. In the general case, for $p$ inks and $N$ solid colorants, the number of independent patterns $I(p)$ required to predict the calibration of the EYNSN model is given by the following equation: 


$$
\begin{aligned}
& N=2^{p} \\
& I(p)=2^{p}+5 \cdot p \cdot 2^{(p-1)}
\end{aligned}
$$

For a CMY print, the number of 2-by-2 independent patterns is $I(3)=68$ and for CMYK this number is $I(4)=176$. According to Bugnon et. al. [12], in the case of CMYK prints, 20 ink spreading curves and therefore 20 calibration halftones are sufficient. For 20 instead of 32 calibration halftones, the number of independent 2-by-2 patterns is therefore 116 instead of 176.

The goal is to predict the reflection spectra of calibration patches with the 2-by-2 dot centering model. We print once the 68 calibration patches of the 2-by-2 dot centering model. Then, for the selected halftoning method, screen shape and screen frequency, we predict with the 2-by-2 dot centering model the reflectance spectra of the corresponding EYNSN calibration patches. This enables establishing the ink spreading curves. One single $n$-value of the Yule-Nielsen modified spectral Neugebauer model is used for both the 2-by-2 dot centering model and for the EYNSN model. By iterating across all possible values, one selects the $n$-value yielding the lowest color difference between predicted and measured reflection spectra. Figure 3 illustrates the dot gain curves corresponding to the ink spreading curves generated by fitting from measured calibration patch reflectance spectra the $50 \%$ surface coverage halftones directly with the EYNSN model (solid lines) and the dot gain curves generated by predicting the $50 \%$ surface coverage halftones with the 2-by-2 prediction model (dashed line). In most cases, the ink spreading curves are very similar.

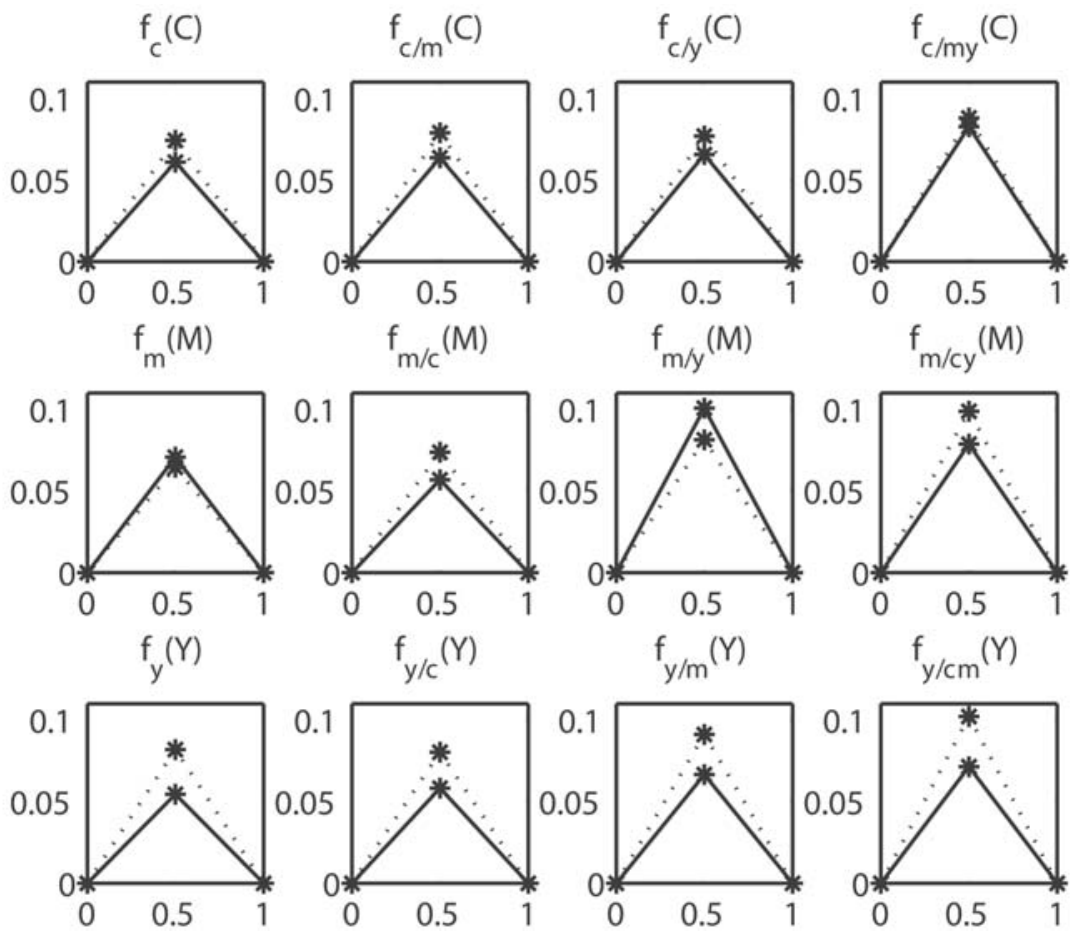

Figure 3: Dot gain curves corresponding to the ink spreading curves fitted with measured halftone calibration spectra (solid lines) and fitted with the halftone calibration patch reflection spectra predicted according to the 2-by-2 model (dashed line). The patches are printed on a Canon Pixma Pro 9500 printer at a screen frequency of 100 lpi and at a resolution of 600 dpi. Dot gain (vertical axis) is defined as effective surface coverage minus nominal surface coverage (horizontal axis).

Figure 4 illustrates the relationship between $n$-value and the spectral prediction accuracy for a test set of 125 CMY color patches at a screen frequency of 1001pi. A mean color prediction deviation of $\triangle \mathrm{E} 94$ is computed 
for each $n$-value between 1 and 7.5. The solid line represents the relationship between prediction accuracy and $n$-value for the original EYNSN model and the dashed line the same relationship for the EYNSN with calibration patch reflectances predicted according to the 2-by-2 model. The best $n$-value for the EYNSN model is 7.5 for an average $\Delta \mathrm{E} 94$ of 0.63 . The best $n$-value for the 2-by-2 prediction of EYNSN calibration patches is 5.1 for an average $\Delta \mathrm{E} 94$ of 0.82 .

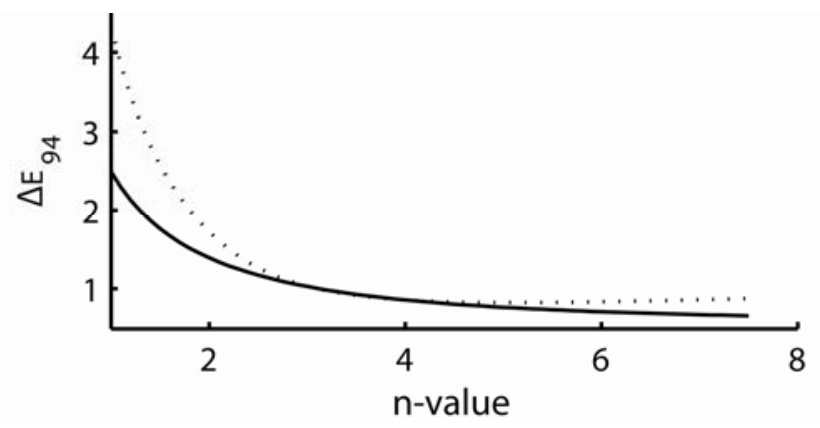

Figure 4: Comparison of the relationship between spectral prediction accuracy and $n$-value for the original EYNSN model (solid line) and for the 2-by-2 calibrated EYNSN model (dashed line) for a print at a screen frequency of 100 lpi.

\section{RESULTS}

We preformed a spectral prediction with both the EYNSN model calibrated by measuring the reflection spectra of the calibration patches and the EYNSN model calibrated with calibration patch reflectances predicted according to the 2-by-2 dot centering model. The experiments were conducted on an ink jet printer (Canon Pixma Pro 9500 at 600 dpi) with a Canon photo pro paper (MP-101). The test samples are printed at three different screen frequencies (75lpi, 100lpi and 125lpi) for a CMY print with coverages varying in steps of 25 percents (125 test patches). In addition, CMYK test patches are printed on the same printer with the same screen frequencies, also for surface coverages varying in steps of 25 percents $\left(5^{4}=625\right.$ test samples). The tables in the Appendix give the mean reflectance prediction error in terms of $\Delta$ E94 values, the maximal prediction error and the $95 \%$ quantile prediction error. For calculating the ink spreading curves, only $50 \%$ nominal coverages in each superposition condition are considered: either predicted by the 2-by-2 dot centering model or measured.

The prediction results shown in the Appendix demonstrate that the measurement based calibration of the EYNSN model is slightly more accurate than the 2-by-2 calibration of the EYNSN model. This is due to the limitation of the 2-by-2 dot centering prediction model, which is always less accurate than the EYNSN model. The EYNSN model, calibrated with 2-by-2 predictions yields substantially the same accuracy as the 2-by-2 model alone.

Table 3 presents the results of predictions on the same CMY test samples when predicting with the 2-by-2 model the ink spreading curves at $25 \%, 50 \%$ and $75 \%$ nominal coverages in each superposition conditions. It clearly appears that the additional 2-by-2 prediction of $25 \%$ and $75 \%$ EYNSN calibration patches does not induce an improved prediction accuracy. In the original EYNSN, a better sampling of the ink spreading curves $(25 \%, 50 \%$ and $75 \%$ nominal surface coverages) reduces the impact of single patch reflectances. When EYNSN calibration patches are predicted with the 2-by-2 model, since more than one 2-by-2 calibration pattern measurement is used to predict each calibration halftone patch reflectances, it is no longer necessary to consider calibration patches at $25 \%, 50 \%$ and $75 \%$ nominal surface coverages. 


\section{CONCLUSIONS}

We propose an extension of the ink spreading enhanced Yule-Nielsen spectral Neugebauer model that enables halftone independent calibration. This is achieved by predicting thanks to the 2-by-2 dot centering model the calibration patches required to create the ink spreading curves. We reduce the calibration effort of the EYNSN model by decreasing the number of calibration patches whose reflectances need to be measured. With 68 measured patch reflectances for cyan, magenta and yellow, respectively 116 measured patch reflectances for cyan, magenta, yellow and black, we are able to predict accurately halftone patches independently of the halftoning method, screen shape and screen frequency. Compared with the original EYNSN model calibration, the calibration relying on the 2-by-2 model introduces only a negligible decrease in prediction accuracy.

The presented approach also provides an important benefit to the users of the 2-by-2 dot centering spectral prediction model. By using the 2-by-2 dot centering model to calibrate the EYNSN model, the number of calibration patch reflectances to be measured is considerably reduced and we still guarantee the same prediction accuracy and the advantages of a halftone-independent algorithm. Moreover, it becomes possible to predict the reflectances of CMYK prints because only 116 calibration 2-by-2 pattern reflectances are needed instead of the 16576 calibration pattern reflectances which would be required by the stand-alone two-by-two dot centering model.

\section{REFERENCES}

[1] R. Balasubramanian, Optimization of the spectral Neugebauer model for printer characterization, J. Electronic Imaging, Vol. 8, No. 2, 156-166, (1999).

[2] D.R. Wyble, R.S. Berns , A Critical Review of Spectral Models Applied to Binary Color Printing, J. Color Res. Appl., Vol. 25, No. 1, 4-19, (2000).

[3] T. Ogasahara, Verification of the Predicting Model and Characteristics of Dye-Based Ink Jet Printer, Journal of Imaging Science and Technology, Vol. 48, No. 2, 130-137 (2004).

[4] R.D. Hersch, F. Crété, Improving the Yule-Nielsen modified spectral Neugebauer model by dot surface coverages depending on the ink superposition conditions, Color Imaging X: Processing, Hardcopy and Applications, SPIE Vol. 5667 (R. Eschbach, G.G. Marcu eds.), 434-445 (2005).

[5] J.A.C. Yule, W.J. Nielsen, The penetration of light into paper and its effect on halftone reproductions, Proc. TAGA, Vol. 3, 65-76, (1951).

[6] J.A.S Viggiano, Modeling the Color of Multi-Colored Halftones, Proc. TAGA, 44-62, (1990).

[7] Shen-ge Wang, Two-by-Two Centering Printer Model with Yule-Nielsen Equation, IS\&T International Conference on Digital Printing Technologies (1998).

[8] Shen-ge Wang, Charles M. Hains, Method of calibrating a digital printer using component test patches and the Yule-Nielsen equation, United States Patent $n^{\circ}$ 5,748,330 (1998).

[9] Shen-ge Wang, Novel Centering Method for Overlapping Correction in Halftoning, Proc IS\&T, 4th Annual Conf., 1994, pp. 482-486

[10] Shen-ge Wang, Feedback for printer Color Calibration, IS\&T International Conference on Digital Printing Technologies (1999).

[11] Shen-ge Wang, Halftone correction systems, United States Patent n ${ }^{\circ} 5854882$ (1998).

[12] T. Bugnon, M. Brichon, R.D. Hersch, Simplified Ink Spreading Equations for CMYK Halftone Prints, Proc. SPIE Vol. 6807, paper 680717-1 to 12 (2008). 


\section{Appendix: Prediction accuracies for ink jet prints}

Table 1: CMY prediction comparison

\begin{tabular}{|c|c|c|c|c|}
\hline $\begin{array}{l}\text { Ink-jet prints (Canon Pixma Pro 9500) } \\
125 \text { CMY test samples }\end{array}$ & $n$-value & $\begin{array}{c}\text { Mean } \\
\Delta E_{94}\end{array}$ & $\begin{array}{c}95 \% \\
\text { quantile }\end{array}$ & $\begin{array}{l}\text { Max } \\
\Delta E_{94}\end{array}$ \\
\hline \multicolumn{5}{|c|}{ Predictions at 75 lpi } \\
\hline EYNSN (20 calib. patches per screen ) & 5 & 0.77 & 1.53 & 2.24 \\
\hline 2-by-2 dot centering model ( 1072 calib. patches) & 3.4 & 0.83 & 1.56 & 1.86 \\
\hline $\begin{array}{l}\text { 2-by-2 prediction of EYNSN calibration patch } \\
\text { reflectances ( } 68 \text { calib. patches) }\end{array}$ & 5.6 & 0.83 & 1.56 & 2.66 \\
\hline \multicolumn{5}{|c|}{ Predictions at 100 lpi } \\
\hline EYNSN (20 calib. patches per screen ) & 7.5 & 0.65 & 1.5 & 1.83 \\
\hline 2-by-2 dot centering model ( 1072 calib. patches) & 4 & 0.81 & 1.62 & 2.54 \\
\hline $\begin{array}{l}\text { 2-by-2 prediction of EYNSN calibration patch } \\
\text { reflectances ( } 68 \text { calib. patches) }\end{array}$ & 5.1 & 0.82 & 1.56 & 2.1 \\
\hline \multicolumn{5}{|c|}{ Predictions at 125 lpi } \\
\hline EYNSN (20 calib. patches per screen ) & 10 & 0.64 & 1.25 & 1.6 \\
\hline 2-by-2 dot centering model ( 1072 calib. patches) & 6.6 & 0.79 & 1.98 & 3.16 \\
\hline $\begin{array}{l}\text { 2-by-2 prediction of EYNSN calibration patch } \\
\text { reflectances ( } 68 \text { calib. patches) }\end{array}$ & 9.7 & 0.81 & 1.89 & 2.44 \\
\hline
\end{tabular}

Table 2: CMYK prediction comparison

\begin{tabular}{|c|c|c|c|c|}
\hline $\begin{array}{c}\text { Ink-jet prints (Canon Pixma Pro 9500) } \\
625 \text { CMYK test samples }\end{array}$ & $n$-value & $\begin{array}{c}\text { Mean } \\
\Delta E_{94}\end{array}$ & $\begin{array}{l}95 \% \\
\text { quantile }\end{array}$ & $\begin{array}{l}\operatorname{Max} \\
\Delta E_{94}\end{array}$ \\
\hline \multicolumn{5}{|c|}{ Predictions at 75 lpi } \\
\hline EYNSN (36 calib. patches per screen ) & 6 & 0.75 & 1.59 & 2.7 \\
\hline $\begin{array}{l}\text { 2-by-2 prediction of EYNSN calibration patch } \\
\text { reflectances ( } 116 \text { calib. patches) }\end{array}$ & 2.4 & 1.15 & 2.64 & 3.71 \\
\hline \multicolumn{5}{|c|}{ Predictions at 100 lpi } \\
\hline EYNSN (36 calib. patches per screen ) & 10 & 1.09 & 2.18 & 3.3 \\
\hline $\begin{array}{l}\text { 2-by-2 prediction of EYNSN calibration patch } \\
\text { reflectances ( } 116 \text { calib. patches) }\end{array}$ & 3.6 & 1.17 & 2.33 & 3.9 \\
\hline \multicolumn{5}{|c|}{ Predictions at 125 lpi } \\
\hline EYNSN (36 calib. patches per screen ) & 10 & 0.75 & 1.8 & 2.3 \\
\hline $\begin{array}{l}\text { 2-by-2 prediction of EYNSN calibration patch } \\
\text { reflectances ( } 116 \text { calib. patches) }\end{array}$ & 3.4 & 1.12 & 2.56 & 3.15 \\
\hline
\end{tabular}




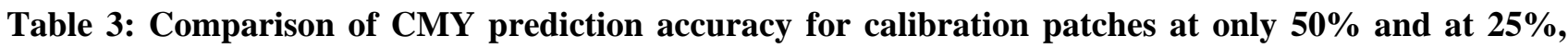
$50 \%, 75 \%$ nominal surface coverages, whose reflectance is predicted with the 2-by-2 model.

\begin{tabular}{|c|c|c|c|c|}
\hline $\begin{array}{r}\text { Ink-jet prints (Canon Pixm } \\
125 \mathrm{CMY} \text { test samp }\end{array}$ & $n$-value & $\begin{array}{c}\text { Mean } \\
\Delta E_{94}\end{array}$ & $\begin{array}{l}95 \% \\
\text { quantile }\end{array}$ & $\begin{array}{l}\operatorname{Max} \\
\Delta E_{94}\end{array}$ \\
\hline \multicolumn{5}{|c|}{ Predictions at 75 lpi } \\
\hline $50 \%$ only & 5.6 & 0.83 & 1.56 & 2.66 \\
\hline $25 \%, 50 \%, 75 \%$ & 4.8 & 0.76 & 1.57 & 2.59 \\
\hline \multicolumn{5}{|c|}{ Predictions at 100 lpi } \\
\hline $50 \%$ only & 5.1 & 0.82 & 1.56 & 2.1 \\
\hline $25 \%, 50 \%, 75 \%$ & 3.3 & 0.96 & 2 & 2.32 \\
\hline \multicolumn{5}{|c|}{ Predictions at 125 lpi } \\
\hline $50 \%$ only & 9.7 & 0.81 & 1.89 & 2.44 \\
\hline $25 \%, 50 \%, 75 \%$ & 4.4 & 0.89 & 1.62 & 2 \\
\hline
\end{tabular}

